\title{
Nucleotide-binding oligomerization domain protein 2 attenuates ER stress-induced cell death in vascular smooth muscle cells
}

\author{
Min-Young Kwon ${ }^{1, \#}$, Narae Hwang ${ }^{1, \#}$, Seon-Jin Lee ${ }^{2} \mathcal{E}$ Su Wol Chung ${ }^{1, *}$ \\ ${ }^{1}$ School of Biological Sciences, University of Ulsan, Ulsan 44610, ${ }^{2}$ Environmental Disease Research Center, Korea Research Institute of \\ Bioscience and Biotechnology, Daejeon 34141, Korea
}

\begin{abstract}
Nucleotide-binding oligomerization domain protein 2 (NOD2), an intracellular pattern recognition receptor, plays important roles in inflammation and cell death. Previously, we have shown that NOD2 is expressed in vascular smooth muscle cells (VSMCs) and that NOD2 deficiency promotes VSMC proliferation, migration, and neointimal formation after vascular injury. However, its role in endoplasmic reticulum (ER) stress-induced cell death in VSMCs remains unclear. Thus, the objective of this study was to evaluate ER stress-induced viability of mouse primary VSMCs. NOD2 deficiency increased ER stress-induced cell death and expression levels of apoptosis mediators (cleaved caspase-3, Bax, and Bak) in VSMCs in the presence of tunicamycin (TM), an ER stress inducer. In contrast, ER stress-induced cell death and expression levels of apoptosis mediators (cleaved caspase-3, Bax, and Bak) were decreased in NOD2-overexpressed VSMCs. We found that the IRE-1 $\alpha$-XBP1 pathway, one of unfolded protein response branches, was decreased in NOD2-deficient VSMCs and reversed in NOD2-overexpressed VSMCs in the presence of TM. Furthermore, NOD2 deficiency reduced the expression of XBP1 target genes such as GRP78, PDI-1, and Herpud1, thus improving cell survival. Taken together, these data suggest that the induction of ER stress through NOD2 expression can protect against TM-induced cell death in VSMCs. These results may contribute to a new paradigm in vascular homeostasis. [BMB Reports 2019; 52(11): 665-670]
\end{abstract}

*Corresponding author. Tel: +82-52-259-1641; Fax: +82-52-259-1694; E-mail: swchung@ulsan.ac.kr

${ }^{\text {"}}$ These authors contributed equally to this work.

https://doi.org/10.5483/BMBRep.2019.52.11.176

Received 12 July 2019, Revised 17 August 2019, Accepted 1 October 2019

Keywords: Cell death, Endoplasmic reticulum stress, NOD2, Tunicamycin, Vascular smooth muscle cells

\section{INTRODUCTION}

A dysfunctional vascular system supply causes nutrient deprivation, hypoxia, and consequently cell death. It is closely associated with protein folding, endoplasmic reticulum (ER) stress (1), and unfolded protein response (UPR) signaling that are readily triggered in vascular cell death (2). The UPR can balance cell survival and cell death and negatively impact vascular development and growth. When ER stress is increased and the protein load in the ER greatly exceeds its folding capacity, cellular dysfunction and cell death often occur. In UPR signaling pathways, IRE- $1 \alpha$ is a key molecule that functions as a mediator of cell fate. IRE- $1 \alpha$ is the most conserved branch of the UPR (3). IRE-1 has a serine/threonine kinase region and ribonuclease region on its cytoplasmic side. Accumulation of unfolded proteins in the ER stimulates IRE1 $\alpha$ oligomerization in ER membranes and autophosphorylation of IRE-1 $\alpha$ (4). Splicing of X-box binding protein 1 (XBP1) is mediated by the RNase activity of IRE-1 to promote proper translation. Splicing by IRE- 1 differs from conventional splicing via spliceosomes as this non-conventional process cuts mature mRNA (5). XBP1 is a transcription factor with a basic leucine zipper domain. It binds to the promoter region of genes related to ER chaperones, ER-associated degradation, ER membrane synthesis, and protein secretion to promote their transcription (6).

NOD2, a member of nucleotide-binding oligomerization domain-like receptors, is an intracellular pathogen sensor that can recognize muramyl dipeptide (7). Clinical and experimental studies have recently revealed a role of NOD2 in cardiovascular diseases by inducing vascular inflammation and affecting the severity of atherosclerosis, the most common pathologic process of coronary artery and cerebrovascular disease (8). NOD2 is localized in inflamed areas of atherosclerotic lesions and overexpressed in endothelial cells, delimiting the lumen of diseased vessels (9). Moreover, the production of NOD2-mediated cytokines such as interleukin (IL)-6, IL-8, and IL-1 $\beta$ can induce vascular inflammation and promote the expansion of lipid-rich necrotic areas (8). The involvement of ER stress and NOD2 in chronic conditions has important implications for understanding the pathogenesis and

ISSN: 1976-670X (electronic edition)

Copyright (C) 2019 by the The Korean Society for Biochemistry and Molecular Biology

(c) This is an open-access article distributed under the terms of the Creative Commons Attribution Non-Commercial License (http://creativecommons.org/licenses/by-nc/4.0) which permits unrestricted non-commercial use, distribution, and reproduction in any medium, provided the original work is properly cited. 
improving the management of these diseases (10). Previous reports have suggested that NOD2 can affect ER stress-induced cell death in VSMCs. The objective of the present study was to determine the mechanism of NOD2-mediated cell death of primary mouse VSMCs and vascular protective effects of NOD2 in ER stress-induced cell death. Our results have potential therapeutic implications for maintaining vascular homeostasis.

\section{RESULTS}

\section{NOD2 deficiency sensitizes TM-induced ER stress cell death in VSMCs}

ER stress-induced cell death was assessed at various times after TM administration in NOD2 ${ }^{+/+}$and $\mathrm{NOD}^{-1-}$ VSMCs using a cell viability Assay. The viability of $\mathrm{NOD}^{-1-}$ VSMCs $(46.5 \%)$ was decreased by TM compared to that of NOD2 ${ }^{+/+}$VSMCs
A

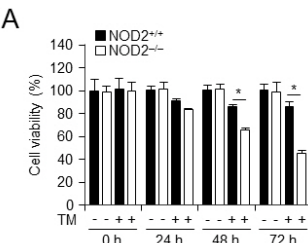

$\mathrm{D}$

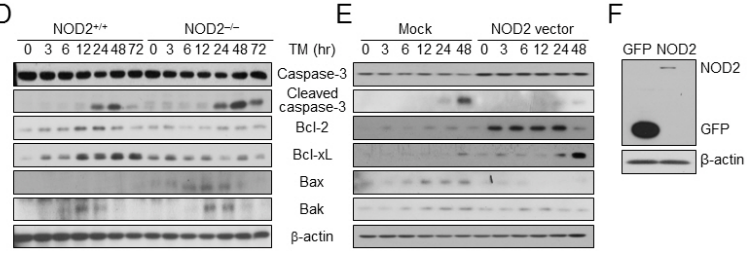

$\mathrm{B}$

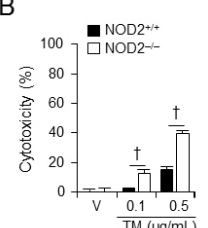

C

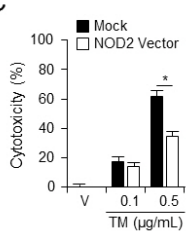

A

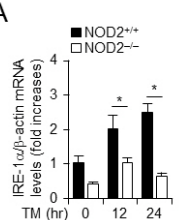

E

Fig. 1. NOD2 deficiency accelerates ER stress induced-cell death. (A) Cell viabilities of NOD2 ${ }^{+/+}$and NOD2 $2^{-1-}$ VSMCs were measured after vehicle or TM $(100 \mathrm{ng} / \mathrm{ml})$ administration. ${ }^{* P}<$ 0.05 , decreased cell viability of $\mathrm{NOD}^{-/-}$vs. NOD2 ${ }^{+/+}$VSMCs in the presence or absence of tunicamycin. Values are presented as mean $\pm \mathrm{SD}(\mathrm{n}=12)$. (B) $\mathrm{NOD}^{+/+}$and $\mathrm{NOD} 2^{-1-}$ VSMCs were treated with vehicle or TM for $48 \mathrm{~h}$. LDH cytotoxicity assay was assessed and represented as a graph. Values are presented as mean $+\mathrm{SD}(\mathrm{n}=12) .{ }^{\top} \mathrm{P}<0.05$, enhanced cell toxicity of $\mathrm{NOD} 2^{-7-}$ vs. NOD2 ${ }^{+1+}$ VSMCs in the presence of TM. (C) Control vector- or NOD2-overexpressed VSMCs were treated with vehicle or TM for $48 \mathrm{~h}$. LDH-cytotoxicity assay was performed. Results are presented as a graph. Values are shown as mean \pm $\mathrm{SD}(\mathrm{n}=12)$. $* \mathrm{P}<0.05$, decreased cell toxicity NOD2overexpressed VSMCs vs, control vector-expressed VSMCs in the presence of TM. (D) Levels of caspase-3, cleaved caspase-3, Bcl-2, $\mathrm{BCl}-\mathrm{xL}, \mathrm{Bax}, \mathrm{Bak}$, and $\beta$-actin were determined at indicated time points after TM $(100 \mathrm{ng} / \mathrm{ml})$ treatment by western blot analysis in NOD $2^{-l-}$ vs. NOD2 ${ }^{+9+}$ VSMCs. Representative blot of three independent experiments. (E) Levels of caspase-3, cleaved caspase-3, $\mathrm{BCl}-2, \mathrm{Bcl}-\mathrm{xL}, \mathrm{Bax}, \mathrm{Bak}$, and $\beta$-actin were determined at indicated time points after TM $(100 \mathrm{ng} / \mathrm{ml})$ treatment by western blotting in control vector or NOD2-overexpressed VSMCs. Representative blots of three independent experiments are shown. (F) NOD2 overexpression was analyzed using FLAG antibody. $\beta$-actin was used as a loading control. NOD2 deficient VSMCs, in vitro cytotoxicity analysis was performed using LDH-cytotoxicity assay. ER stress-induced cell death was enhanced in NOD2 $2^{-1-}$ VSMCs compared to that in NOD2 $2^{+/+}$VSMCs at $48 \mathrm{~h}$ after TM treatment (Fig. 1B). However, NOD2-overexpressed VSMCs showed decreased cell death in response to TM (Fig. 1C). These data suggest that NOD2-deficient VSMCs are highly susceptible to TM-induced cell death. Whether NOD2 deficiency and overexpression affected the expression of apoptosis-related proteins such as caspase-3, Bcl-2, Bcl-xL, Bak, and Bax at various time points after TM treatment was also investigated. Results are shown in Fig. 1C. In NOD2 $2^{-1-}$ VSMCs (NOD2 deficiency) levels of cleaved caspase-3, Bax, and Bak were increased whereas levels of Bcl-2, Bcl-2, and Bcl-xL were decreased after TM treatment compared to those in NOD2 $2^{+/+}$VSMCs. Next, levels of apoptosis-related proteins in the control and NOD2-overexpressing NOD2 $2^{+/+}$VSMCs were investigated. Protein levels of cleaved caspase- 3 and pro-apoptotic members of Bcl-2, Bax, and Bak were decreased while Bcl-2 and Bcl-xL levels were enhanced in NOD2-overexpressed VSMCs (Fig. 1D). These data suggest that NOD2 can regulate ER stress-induced cell death.

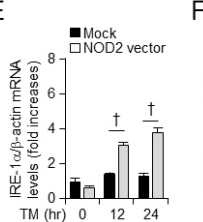

B
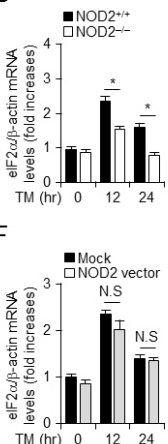

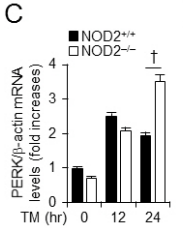

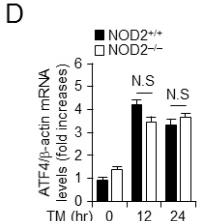

$\mathrm{H}$

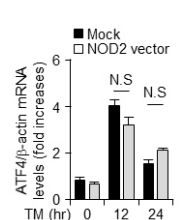

(85.1\%) (Fig. 1A). To verify ER stress-induced cell death in

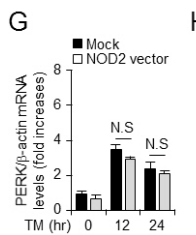

Fig. 2. NOD2 expression modulates TM-induced ER stress by regulating mRNA levels of IRE- $1 \alpha$ in vascular smooth muscle cells. mRNA levels of ER stress-related genes at indicated times in the presence or absence of TM treatment of $\mathrm{NOD}^{+/+}$and $\mathrm{NOD} 2^{-l-}$ VSMCs were determined. Quantitative real-time RT-PCR was performed to assess mRNA levels of IRE-1 $\alpha$ (A), elF2 $\alpha$ (B), PERK (C), and ATF4 (D) in NOD2 ${ }^{+1+}$ and NOD2 ${ }^{-1-}$ VSMCs. *P $<0.05$ down-regulation of gene expression in $\mathrm{NOD}^{-1-} \mathrm{vs}$. $\mathrm{NOD}^{+1+}$ VSMCs. ${ }^{+} \mathrm{P}<0.05$, up-regulation of gene expression in $\mathrm{NOD}^{-1-}$ vs. NOD2 ${ }^{+/+}$VSMCs. After transfection with NOD2 overexpression or mock vector in the presence and absence of TM treatment, mRNA levels of ER stress-induced factor of IRE-1 $\alpha$ $(\mathrm{E})$, elF2 $\alpha(\mathrm{F})$, PERK $(\mathrm{G})$, and ATF4 $(\mathrm{H})$ in primary mouse smooth muscle cells at indicated time points were measured by quantitative real-time PCR analysis. ${ }^{\dagger} \mathrm{P}<0.05$, up-regulation of gene expression in NOD2 over-expressing vs. control. For all real-time RT-PCR analyses, mouse $\beta$-actin was used as a control for normalization. Values are presented as mean $\pm S D(n=3)$. 


\section{NOD2 deficiency enhances TM-induced IRE-1 $\alpha$ mRNA expression in mouse VSMCs}

We found that NOD2 expression level was a crucial factor in TM-mediated cell death in vitro. Next, we investigated whether NOD2 could stimulate TM-induced ER stress signal transduction in VSMCs. Transcription levels of ER stress-related proteins such as IRE- $1 \alpha$, elF- $2 \alpha$, PERK, and ATF4 were examined by real-time PCR after inducing ER stress with TM $(100 \mathrm{ng} / \mathrm{ml})$ in $\mathrm{NOD}^{-1-}$ and littermate VSMCs for different times (Figs. 2A-2D). When NOD2 ${ }^{-l-}$ VSMCs were treated with TM, IRE- $1 \alpha$ and elF-2 $\alpha$ expression levels were decreased (Figs. 2A and 2B). In contrast, PERK mRNA level was increased in NOD2-deficient VSMCs (Fig. 2C), although ATF4 mRNA level was not significantly affected by TM treatment compared to that in control littermates (Fig. 2D). We hypothesized that the NOD2 expression level was a critical factor in TM-induced ER stress. To evaluate whether gain of NOD2 function could influence TM-induced IRE- $1 \alpha$ or elF- $2 \alpha$ signal transduction, we also transiently overexpressed NOD2 with pcDNA3.1GFP-NOD2 vector in VSMCs. Real-time PCR was performed to examine levels of mRNA encoding IRE- $1 \alpha$, elF- $2 \alpha$, PERK, and ATF4 to determine whether they were also affected by TM-induced ER stress. Interestingly, mRNA levels of IRE-1 $\alpha$ were significantly increased upon treatment with $T M$ in NOD2-overexpressed VSMCs for $24 \mathrm{~h}$ (Fig. 2E), while mRNA levels of elF-2 $\alpha$ (Fig. 2F), PERK (Fig. 2G), or ATF4 (Fig. 2H) were unaffected by TM-induced ER stress. These results show

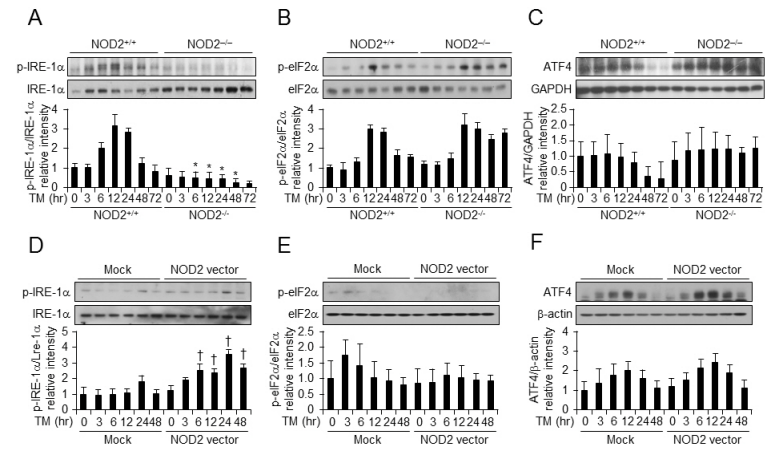

Fig. 3. NOD2 expression regulates translational levels of IRE- $1 \alpha$ in vascular smooth muscle cells. Western blot analysis at different time points after vehicle $(\mathrm{V})$ or TM $(100 \mathrm{ng} / \mathrm{ml})$ treatment of NOD2 vs $\mathrm{NOD}^{+/+}$VSMCs. Western blot analysis was performed to detect levels of phospho-IRE1 $\alpha$ (A), p-eIF2 $\alpha$ (B), and ATF4 (C) normalized to IRE- $1 \alpha$, elF $2 \alpha$, and GAPDH. The control vector or NOD2 expression vector was introduced into $\mathrm{NOD}^{+/+}$VSMCs. Western blot analysis was performed for XBP1s after vehicle (V) or TM $(100 \mathrm{ng} / \mathrm{ml})$ treatment. Phospho-IRE1 $\alpha$ (D), p-elF2 $\alpha$ (E), and ATF4 (F) levels were normalized to IRE- $1 \alpha$, elF2 $\alpha$, and $\beta$-actin levels determined by western blot analysis. All data are shown as mean $\pm \mathrm{SD}$ of three independent experiments. $* \mathrm{P}<0.05$ down-regulation of gene expression in $\mathrm{NOD}^{-l-}$ vs. $\mathrm{NOD} 2^{+/ t}$ VSMCs. ${ }^{+} \mathrm{P}<0.05$, up-regulation of gene expression in NOD2 overexpressing vs. control. $\beta$-actin was used as a loading control. that NOD2 expression is related to the transcriptional level of IRE- $1 \alpha$, an ER stress-induced gene in mouse VSMCs.

\section{Depletion of NOD2 accelerates the translation level of IRE-1 $\alpha$ in TM-induced ER stress}

To assess the role of NOD2 in TM-induced ER stress signaling, we isolated VSMCs from NOD2 ${ }^{-1-}$ mice and their littermate controls. We also evaluated whether NOD2 expression activated the ER stress pathway following TM treatment compared to $\mathrm{NOD}^{-/-}$and NOD2 ${ }^{+/+}$VSMCs. VSMCs showed increased post-translational activation of ER stress-related markers including phospho-elF $2 \alpha$, phospho-IRE- $1 \alpha$, and ATF4 by TM treatment for indicated times (Figs. 3A-3C). To demonstrate that the gain of NOD2 function could influence TM-mediated ER stress activation, we induced NOD2 vector-mediated NOD2 overexpression in VSMCs for different times. As shown in Fig. 3D-3F, NOD2 vector-mediated NOD2 overexpression in VSMCs resulted in an increase in ER stress signaling with high phosphorylation levels of IRE-1 $\alpha$. GPER expression was increased, although phosphorylation of elF $2 \alpha$ or ATF 4 was not.

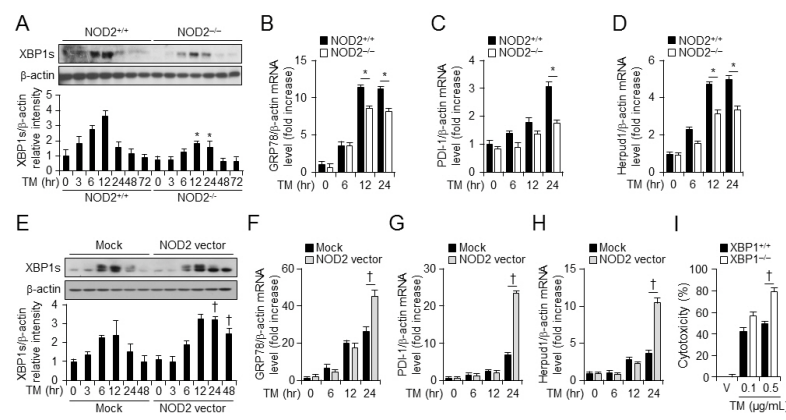

Fig. 4. NOD2 induces the expression of $X B P$ and $E R$ chaperone molecules. (A) Western blotting for XBP1s was performed at indicated time points after TM $(100 \mathrm{ng} / \mathrm{ml})$ treatment of NOD2 vs. NOD2 ${ }^{+/+}$VSMCs. $\beta$-actin was used as a loading control. Quantitative real-time RT-PCR was performed to assess mRNA levels of (B) GRP78, (C) PDI-1, and (D) Herpud1 in NOD2 ${ }^{+1+}$ and NOD2 ${ }^{-1-}$ VSMCs. The control vector (pCDNA3/GFP) or NOD2 expression vector was introduced into $\mathrm{NOD}^{+/+}$VSMCs. ${ }^{* P}<$ 0.05 , down-regulation of gene expression in $\mathrm{NOD} 2^{-1-}$ vs. NOD2 ${ }^{+/+}$VSMCs. (E) Western blotting was performed for XBP1s after vehicle $(\mathrm{V})$ or TM $(100 \mathrm{ng} / \mathrm{ml})$ treatment. $\beta$-Actin was used as a control for normalization. Mock or NOD2-expressing cells after vehicle $(\mathrm{V})$ or tunicamycin $(100 \mathrm{ng} / \mathrm{ml})$ treatment for indicated time. Quantitative real-time RT-PCR was performed to assess mRNA levels of (F) GRP78, (G) PDI-1, and (H) Herpud1 in primary mouse NOD2 ${ }^{+/+}$VSMCs. Representative blot of three independent experiments. ${ }^{\dagger} \mathrm{P}<0.05$, up-regulation of gene expression in NOD2-overexpressed vs. control cells. For all real-time RT-PCR analyses, mouse $\beta$-actin was used as a control for normalization. Values are presented as mean $\pm \mathrm{SD}(\mathrm{n}=3)$. (I) $\mathrm{XBP} 1^{+/+}$and $\mathrm{XBP} 1^{-1-}$ MEFs were treated with vehicle or TM for $48 \mathrm{~h}$. $\mathrm{LDH}$-cytotoxicity was assessed and presented as a graph. Values are shown as mean $\pm \mathrm{SD}(\mathrm{n}=12) .{ }^{\dagger} \mathrm{P}<0.05$, enhanced cell toxicity of $\mathrm{XBP}^{-l-}$ vs. XBP1 ${ }^{+1+}$ MEFs in the presence of TM. 


\section{NOD2 regulates XBP1 and UPR gene expression}

We examined whether NOD2 expression was related to ER stress factor IRE1 $\alpha$. Results are shown in Fig. 3. NOD2 reduced ER stress by regulating XBP1s and UPR gene expression as well as GRP78, PDI-1, and Herpud1 expression. Total protein was harvested at various time points after TM treatment from $\mathrm{NOD}_{2}^{+/+}$and $\mathrm{NOD} 2^{-/-}$VSMCs. Protein levels of XBP1s began to increase by $3 \mathrm{~h}$. A significant increase of XBP1s was evident after $12 \mathrm{~h}$ of TM treatment in NOD2 ${ }^{+/+}$VSMCs (Fig. 4A). However, protein levels of XBP1s were decreased in NOD2 $2^{-1-}$ VSMCs in the presence of TM (Fig. 4A). Overexpression of NOD2 increased XBP1s protein levels compared to overexpression of a control vector (Fig. 4E). To investigate mRNA levels of UPR genes, total RNAs were harvested at 6,12 , and $24 \mathrm{~h}$ after TM treatment from $\mathrm{NOD}^{+/+}$and $\mathrm{NOD}^{-1-}$ VSMCs or control and NOD2overexpressed VSMCs. mRNA levels of UPR genes were then analyzed by quantitative real-time RT-PCR. mRNA levels of GRP78 (Fig. 4B), PDI-1 (Fig. 4C), and Herpud1 (Fig. 4D) were decreased in NOD2 ${ }^{-1-}$ VSMCs compared to those in $\mathrm{NOD}^{+/+}$VSMCs. Moreover, mRNA levels of these genes were induced in NOD2-overexpressed VSMCs (Figs. 4F-4H). To confirm that downregulation of XBP1 in NOD2 deficient VSMCs could lead to enhanced ER stress-induced cell death, we performed $\mathrm{LDH}$-cytotoxicity assay in $\mathrm{XBP}^{+/+}$and $\mathrm{XBP}^{-1-}$ mouse embryonic fibroblasts (MEFs). Tunicamycin-induced cell death was enhanced in $\mathrm{XBP}^{-1-}$ MEFs compared to that in $\mathrm{XBP}^{+/+}$MEFs (Fig. 4I). These data suggest that NOD2 can increase UPR gene expression during TM-induced ER stress in VSMCs.

\section{DISCUSSION}

VSMCs play important roles not only in physiological functions such as vasoconstriction, vasodilatation, and extracellular matrix production of blood vessels, but also in the pathogenesis of vascular diseases, particularly atherosclerosis and hypertension (11). In healthy adults, VSMCs are quiescent. However, after blood vessel injury, they undergo phenotypic modulation from the contractile phenotype to the synthetic phenotype characterized by high migration, proliferation, and proteosynthesis (11). This behavior of VSMCs can lead to stenosis or obliteration of the vascular lumen. Recently, prolonged ER stress in atherosclerotic lesions has been found to be an important contributor to proatherogenic progression (12). However, mechanisms of ER stress-mediated apoptosis in VSMCs have not been widely studied compared to those in macrophages or endothelial cells. In this study, we confirmed that TM as an ER stress inducer could promote apoptosis in VSMCs (Fig. 1).

Pattern recognition receptors such as Toll-like receptors play key roles in the pathogenesis of atherosclerosis. They are involved in inflammatory processes and ER stress-induced cell death (13-15). However, functional roles or mechanisms of NOD2, an intracellular PRR, in ER stress-induced cell death of
VSMCs have not been reported yet. Using NOD2-deficient VSMCs, we demonstrate that NOD2 plays a crucial role in regulating vascular homeostasis by attenuating ER stress-induced smooth muscle cell mortality. NOD2 may regulate ER stress-induced cell death by several distinct mechanisms. In Fig. 1, ER stress-induced cell death and apoptosis-associated molecules such as cleaved caspase-3, Bax, and Bad were increased in NOD2-deficient VSMCs compared to in wild-type cells, whereas $\mathrm{BCl}-2$ or $\mathrm{BCl}-\mathrm{xL}$ was not. Overexpression of NOD2 in wild-type VSMCs reversed these effects in NOD2-deficient VSMCs.

Our studies showed that NOD2 might be an important target for treating chronically vascular diseases such as atherosclerosis and retinopathy. Recent reports have indicated that the involvement of NOD2 in orchestrating this inflammatory branch of the UPR has broad implications as ER stress is linked to several inflammatory diseases, including atherosclerosis and Crohn's disease $(16,17)$. Crohn's disease is of particular interest because certain alleles in the human NOD2 gene are major risk factors for developing this inflammatory disorder (17). Accumulating evidence has shown that altered cell function in Crohn's disease occurs because of ER stress. Our study showed that depletion of NOD2 increased VSMC mortality through ER stress-related apoptosis.

Thus, NOD2 might be modulated by protective roles of TM-induced ER stress signals on VSMCs to enhance vascular homeostasis. As shown in Fig. 1, NOD2 deficiency may be related to enhanced apoptosis of VSMCs. TM-induced apoptosis including the activation of caspase-3, Bax, and Bak was examined in VSMCs in response to depletion of the NOD2 gene and NOD2 overexpression to determine the role of NOD2 in ER stress-induced cell death.

In the current study, we found that NOD2 deficiency promoted the down-regulation of IRE $1 \alpha$ expression in VSMCs. Moreover, our results revealed that NOD2 deficiency or overexpression is related to the mRNA levels of ER stress signal proteins such as IRE- $1 \alpha$ rather than elF2 $\alpha$, PERK, or ATF4. We also confirmed that NOD2 depleted or overexpressed cells had typical characteristics of UPR, particularly splicing of XBP1 protein and phosphorylation of elF2 $\alpha$ and ATF4. These findings suggest that NOD2 signaling pathway activated ER stress following exposure to TM and TM-induced enhancement of ER stress via IRE- $1 \alpha$ expression might be related to smooth muscle cell death.

IRE- $1 \alpha$ has been found to be the most conserved factor in the UPR recently (18). IRE $1 \alpha$ dimerizes and transphosphorylates itself in the UPR. This mediates the activation of endoribonuclease activity in IRE- $1 \alpha$, leading to splicing of XBP1 mRNA in the cytosol (18). XBP1s, the protein translated from the spliced XBP1 mRNA, is localized in the nucleus. It can induce the expression of proteins involved in ER-associated degradation, chaperones, and lipid synthesis (19). Activated IRE-1 also mediates the rapid degradation of a specific subset of mRNAs known as regulated IRE-1-dependent decay 
implicated in cell death (19). Furthermore, we confirmed that XBP1 deficient MEFs were more susceptible to tunicamycininduced cell death than control MEFs (Fig. 4I).

In conclusion, NOD2 may protect against TM-induced cell death and maintain VSMC homeostasis in vitro. Modulating NOD2 status to regulate TM-induced cell mortality has potential therapeutic effects in VSMCs. Understanding the relationship between NOD2 expression and induction of ER stress caused by TM may result in improved treatments for VSMCs. We found that NOD2 signal transduction could activate ER stress through activation of IRE- $1 \alpha$. Determining the exact mechanism of regulation and functions of NOD2 may lead to the development of more effective therapies for maintaining vascular homeostasis.

\section{MATERIALS AND METHODS}

\section{Cell culture and reagents}

Primary $\mathrm{NOD}^{+/+}$and $\mathrm{NOD} 2^{-/-}$VSMCs from mice were obtained by collagenase and elastase digestion of aortas as described previously (9). $\mathrm{XBP} 1^{+/+}$and $\mathrm{XBP} 1^{-1-}$ mouse embryonic fibroblasts (MEFs) were kindly provided by $\mathrm{SH}$ Back (Department of Biological Sciences, University of Ulsan, Ulsan, Korea). MEFs were cultured in Dulbecco Modified Eagle Medium (Life Technologies, Grand Island, NY, USA) supplemented with $10 \%$ FBS, penicillin, and streptomycin. TM and other reagents were obtained from Sigma-Aldrich (St. Louis, MO, USA).

\section{Cell viability and cell toxicity assays}

Cell viability was determined by the MTS assay using an AQueous One Solution Cell Proliferation Assay kit (Promega, Madison, WI, USA). Cells were treated with TM for different times. After incubation with MTS solution, absorbance at 490 $\mathrm{nm}$ was measured using a SpectraMax M2 microplate reader (Molecular Devices, Sunnyvale, CA, USA) to calculate cell survival percentages normalized to those of the control group. Lactate dehydrogenase (LDH)-cytotoxicity fluorometric assay was used to assess cytotoxicities (BioVision, Milpitas, CA, USA). The assay was performed according to the manufacturer's instructions.

\section{Transfection}

Plasmid pcDNA4HisMax-mNOD2 was kindly provided by Michael P. Davey (Oregon Health and Sciences University, Portland, OR, USA). Mouse NOD2 cDNA was cloned into the pcDNA3-GFP vector. VSMCs were transfected with the pcDNA3/GFP-NOD2 vector for $24 \mathrm{~h}$ using Lipofectamine 2000 (Invitrogen, Carlsbad, CA, USA) based on the manufacturer's protocol and recovered in DMEM containing $20 \%$ fetal bovine serum, penicillin, and streptomycin. After recovering, stably transfected cells were selected based on their resistance to neomycin for two weeks. Neomycin-resistant cells containing NOD2 cDNA plasmid compared to cells containing the control vector pcDNA-GFP were used for western blot analysis using a GFP antibody (Santa Cruz Biotechnology, Dallas, TX, USA).

\section{Western immunoblotting}

Western immunoblotting was performed as previously described (9). Briefly, cells were harvested using RIPA buffer with protease inhibitors (Roche Applied Science, Mannheim, Germany). Protein concentrations were determined using a BCA protein assay kit (Thermo Fisher Scientific, Waltham, MA, USA). Samples were resolved with SDS-polyacrylamide gels and transferred to PVDF membranes (Bio-Rad, Hercules, CA, USA) overnight. Transferred membranes were hybridized with various antibodies including elF2 $\alpha$, phosphor-elF $2 \alpha, \mathrm{BCl}-\mathrm{xL}$, Bcl-2, Bak, GAPDH (Santa Cruz Biotechnology), IRE1 $\alpha$, caspase-3, cleaved caspase-3 (Cell Signaling Technology, Danvers, MA), ATF4 (Proteintech, Rosemont, IL, USA), XBP1, phosphorylated IRE $1 \alpha\left(\right.$ abcam $^{\circledR}$, Cambridge, UK), and $\beta$-actin (Sigma-Aldrich). These blots were stained with horseradish peroxidase-conjugated IgG and visualized with a SuperSignal West Pico Chemiluminescent Substrate (Pierce, Rockford, IL, USA).

\section{Quantitative real-time RT-PCR}

Total RNAs were isolated from VSMCs and tissues using Trizol reagent (Invitrogen) and reverse transcribed to cDNAs using a SuperScript ${ }^{\mathrm{TM}}$ III First-Strand Synthesis System (Invitrogen). Real-time quantitative PCR was conducted using iQ SYBR Green Supermix (Bio-Rad). Primer sequences were as follows: mouse PERK (5'-CCG TGA CCC ATC TGC ACT AAT-3' and $5^{\prime}$-CAT AAA TGG CGA CCC AGC TT-3'), IRE- $1 \alpha\left(5^{\prime}\right.$-CAG ATC TGC GCA AAT TCA GA-3' and 5'-CTC CAT GGC TTG GTA GGT GT-3'), ATF4 (5'-ATG GCC GGC TAT GGA TGA T-3' and $5^{\prime}$-GAA GTC AAA CTC TTT CAG ATC CAT T- $3^{\prime}$ ), elF $2 \alpha$ (5'-ACT TCG GGA TTC ACA CAT CC-3' and 5'-GCC AAT TCG GAT CAG TTT GT-3'), GRP78 (5'-CAT GGT TCT CAC TAA AAT GAA AGG-3' and 5'-CTG GTA CAG TAA CAA CTG-3'), PDI-1 (5'-CAA GAT CAA GCC CCA CCT GAT-3' and 5'-AGT TCG CCC CAA CCA GTA CTT-3'), Herpud1 (5'-AGC AGC CGG ACA ACT CTA AT-3' and 5'-CTT GGA AAG TCT GCT GGA CA-3'), $\beta$-actin (5'-CTC CAT CAT GAA GTG TGA CG-3' and 5'-ATA CTC CTG CTT GCT GAT CC-3'). cDNA was amplified for $10 \mathrm{~min}$ at $95^{\circ} \mathrm{C}$, followed by 40 cycles of $15 \mathrm{~s}$ at $95^{\circ} \mathrm{C}$ and 1 min at $60^{\circ} \mathrm{C}$.

\section{Statistical analysis}

Data are presented as mean \pm SD. To compare two groups, Student's two-tailed unpaired $t$ test was used. To compare more than two groups and for multiple comparisons, analysis of variance (ANOVA) was used. Statistically significant differences were accepted at $\mathrm{P}<0.05$.

\section{ACKNOWLEDGEMENTS}

This research was supported by a grant (NRF-2014R1A6A 
1030318 to S.W.C.) of the Basic Science Research Program through the National Research Foundation of Korea (NRF), funded by the Ministry of Education. This research was also supported by a grant from the KRIBB Research Initiative Program.

\section{CONFLICTS OF INTEREST}

The authors have no conflicting interests.

\section{REFERENCES}

1. Binet $F$ and Sapieha $P$ (2015) ER Stress and Angiogenesis. Cell Metab 22, 560-575

2. Scull CM and Tabas I (2011) Mechanisms of ER stressinduced apoptosis in atherosclerosis. Arterioscler Thromb Vasc Biol 31, 2792-2797

3. Jiang D, Niwa M and Koong AC (2015) Targeting the IRE1 $\alpha$-XBP1 branch of the unfolded protein response in human diseases. Semin Cancer Biol 33, 48-56

4. Li H, Korennykh AV, Behrman SL and Walter P (2010) Mammalian endoplasmic reticulum stress sensor IRE1 signals by dynamic clustering. Proc Natl Acad Sci U S A $107,16113-16118$

5. Hirota $M$, Kitagaki $M$, Itagaki $H$ and Aiba $S$ (2006) Quantitative measurement of spliced XBP1 mRNA as an indicator of endoplasmic reticulum stress. J Toxicol Sci 31, 149-156

6. Yoshida $H$, Matsui $T$, Yamamoto A et al (2001) XBP1 mRNA is induced by ATF6 and spliced by IRE1 in response to ER stress to produce a highly active transcription factor. Cell 107, 881-891

7. Franchi L, McDonald C, Kanneganti TD et al (2006) Nucleotide-binding oligomerization domain-like receptors: intracellular pattern recognition molecules for pathogen detection and host defense. J Immunol 177, 3507-3513

8. Johansson ME, Zhang $X Y$, Edfeldt $K$ et al (2014) Innate immune receptor NOD2 promotes vascular inflammation and formation of lipid-rich necrotic cores in hypercholesterolemic mice. Eur J Immunol 44, 3081-3092

9. Kwon MY, Liu X, Lee SJ et al (2011) Nucleotide-binding oligomerization domain protein 2 deficiency enhances neointimal formation in response to vascular injury. Arterioscler Thromb Vasc Biol 31(11), 2441-24417

10. Keestra-Gounder AM, Byndloss MX, Seyffert $N$ et al (2016) NOD1 and NOD2 signalling links ER stress with inflammation. Nature 532, 394-397

11. Grootaert MOJ, Moulis M, Roth L et al (2018) Vascular smooth muscle cell death, autophagy and senescence in atherosclerosis. Cardiovasc Res 114, 622-634.

12. Chistiakov DA, Sobenin IA, Orekhov AN and Bobryshev YV (2014) Role of endoplasmic reticulum stress in atherosclerosis and diabetic macrovascular complications. Biomed Res Int 2014, 610140

13. Hansen MK, Kassiteridi C and Monaco C (2013) Toll-like receptors in atherosclerosis. Int J Mol Sci 14, 14008-14023

14. Lim J, Kim HK, Kim SH et al (2017) Caspase-2 mediates triglyceride (TG)-induced macrophage cell death. BMB Rep 50, 510-515

15. Maeng HJ, Song JH, Kim GT et al (2017) Celecoxibmediated activation of endoplasmic reticulum stress induces de novo ceramide biosynthesis and apoptosis in hepatoma HepG2 cells mobilization. BMB Rep 50, 144-149.

16. Roshan $\mathrm{MH}$, Tambo A and Pace NP (2016) The Role of TLR2, TLR4, and TLR9 in the pathogenesis of atherosclerosis. Int J Inflam 2016, 1-11

17. Fritz T, Niederreiter L, Adolph T et al (2011) Crohn's disease: NOD2, autophagy and ER stress converge. Gut 60, 1580-1588

18. Chalmers F, van Lith M, Sweeney B et al (2017) Inhibition of IRE1 $\alpha$-mediated XBP1 mRNA cleavage by XBP1 reveals a novel regulatory process during the unfolded protein response. Wellcome Open Res 2, 1-25

19. Coelho DS and Domingos PM (2014) Physiological roles of regulated Ire1 dependent decay. Front Genet 5, 1-19 